I. Mühlhauser · J. Kasper · G. Meyer $\cdot$

Federation of European Nurses in Diabetes

\title{
Understanding of diabetes prevention studies: questionnaire survey of professionals in diabetes care
}

Received: 11 January 2006 / Accepted: 28 March 2006 / Published online: 23 May 2006

C) Springer-Verlag 2006

\begin{abstract}
Aims/hypothesis: Diabetes prevention studies have reported reductions of diabetes risk by up to $60 \%$. Since the underlying metabolic changes are small, the clinical significance of this effect may be overestimated. The present survey explores the extent to which different formats of presenting study results may influence diabetes healthcare professionals' perceptions of the importance of intervention effects on diabetes risk. Subjects, materials and methods: Participants of three European diabetes conferences (160 nurse educators, 112 physicians, 27 other professionals) were presented with a questionnaire that included nine items, in which results from three diabetes prevention studies were presented in different ways. Results: Participation rate was $96 \%$. Effects were interpreted as important or very important by $92 \%(255 / 276)$ when results were presented as proportions of subjects with diabetes $(14 \%$ intervention group, $29 \%$ control group), by $87 \%(248 / 285)$ when results were communicated as a risk reduction of $57 \%$, by $39 \%(110 / 284)$ when the corresponding fasting plasma glucose values were presented (mean difference $0.3 \mathrm{mmol} / \mathrm{l}$ ), and by $18 \%$ $(52 / 283)$ when glycosylated haemoglobin values were used (6.0 vs $6.1 \%)$. Corresponding results of the three diabetes prevention studies were rated as being of identical importance by only 23,13 and $16 \%$ of participants,
\end{abstract}

Details on Federation of European Nurses in Diabetes (FEND) contribution, see Appendix

Electronic Supplementary Material Supplementary material is available in the online version of this article at http://www.10.1007/ s00125-006-0290-8

I. Mühlhauser $(\square) \cdot$ J. Kasper · G. Meyer

Universität Hamburg,

Martin-Luther-King Platz 6,

D-20146 Hamburg, Germany

e-mail: Ingrid Muehlhauser@uni-hamburg.de

Tel.: +49-40- $\overline{42} 8383988$

Fax: $+49-40-428383732$ respectively. Conclusions and interpretation: Healthcare professionals rate the benefit of preventive interventions substantially higher when changes in diabetes risk are communicated rather than related glycaemic parameters. Transformation of continuous metabolic data into diagnostic categories may impair understanding of study effects.

Keywords Communication - Comprehension - Diabetes mellitus type 2/prevention \& control · Evidence-based medicine/standards · Questionnaires · Risk

Abbreviations ESM: Electronic supplementary material . RRR: relative risk reduction - UKPDS: United Kingdom Prospective Diabetes Study · WHI: Women's Health Initiative

\section{Introduction}

Healthcare professionals are increasingly asked to communicate research results to patients and consumers $[1,2]$. This requires translation of study results into clear and understandable information [3]. Framing of data is a wellrecognised cause of misconceptions about the efficacy of health interventions by physicians [4-6], healthcare decision-makers [7], and patients [8]. This is particularly relevant for preventive medicine [9].

Recently, several randomised controlled trials on the primary prevention of diabetes have been published [10 13]. Since then a reduction of diabetes by more than $50 \%$ has been publicly claimed and large-scale population interventions are being promoted $[14,15]$.

However, publications of the prevention studies do not always report important outcome data, and framing of results may be a major problem, possibly leading to overestimation of the clinical significance of the intervention effect on diabetes risk [16].

The aim of the present study is to survey the understanding of different formats of data presentation of results 
of preventive diabetes studies among diabetes healthcare professionals.

\section{Subjects, materials and methods}

\section{Participants}

The survey sample comprised the participants of three annual diabetes meetings: (1 and 2) the Federation of European Nurses in Diabetes $(n=162)$ and Primary Care Diabetes Europe (primarily physicians with a special interest in diabetes, $n=49$ ), both held in September 2005 in Athens on the occasion of the 41st Annual Meeting of the European Association for the Study of Diabetes; (3) the Postgraduate Course in Clinical Diabetology $(n=101)$, primarily physicians specialising in diabetes, held in November 2005 at the University of Jena, Germany.

\section{Questionnaire}

Results of four publications formed the basis of the questionnaires $[10,12,17,18]$. Articles were selected according to their clinical and public impact and their appropriateness for data extraction for different presentations of study results. Two studies explicitly targeted primary prevention of diabetes. Results of the lifestyle intervention arms were used $[10,12]$. The third study, the Women's Health Initiative (WHI), described the effects of combined oestrogen plus progestin or placebo on metabolic parameters and the self-reported incidence of diabetes over more than 5 years of follow-up [17]. The fourth article was the main publication of the United Kingdom Prospective Diabetes Study (UKPDS), a landmark study on secondary prevention in persons with newly diagnosed type 2 diabetes [18]. Ten items were developed. The questionnaire with an explanation on how alternative presentations were derived is available as electronic supplementary material (ESM). Corresponding study results were used in items 1 and 4 [10], items 2, 3, 5 and 9 [17], and items 7 and 8 [12]; item 6 was not based on real data, but displayed relative risk reductions that were comparable to item 1 but at lower absolute risks. In item 10 , the main results of the UKPDS on the primary endpoint were used to evaluate understanding of natural frequencies [18]. In some items, there were minor changes of the original data to avoid immediate recognition of related study results, e.g. study duration 3.3 years instead of 3 years. Almost identical wording was used in all items: 'Looking at this result (these results, this graph), what is your impression about the effect of this (preventive) intervention on diabetes risk?' Participants were asked to mark their ratings as either 'very important $(++)$ ', 'important $(+)$ ', 'not very important $(-)$ ' or 'not important at all $(--)^{\prime}$. The first draft of the questionnaire was pilot tested with 20 diabetes healthcare professionals from various European countries.

\section{Assumptions}

Based upon a vast literature on framing of data [3-8] and the results of the pilot study, the following assumptions were made. Effect sizes are rated highest when presented as relative risk reduction (RRR) with higher numerical RRRs being more important than lower values (item 1 vs item 3 ). Effect sizes are perceived to be of decreasing importance when results are presented as proportions of subjects with a diagnosis of diabetes (item 8) or as graphs with truncated scales (item 5), and of lowest importance when (1) a complete scale from 0.0 to 1.0 is used (item 9), (2) results are presented as proportions of subjects remaining free of disease (item 2) or (3) when the actual blood glucose (item 4) or $\mathrm{HbA}_{1 \mathrm{c}}$ values are presented (item 7). Identical relative differences are rated of lower importance when absolute risks are low (item 6).

\section{Data collection and analysis}

The English version of the questionnaire was distributed to all conference participants by one or two of the authors. An identical standard introduction was used. Participants were asked to rate the ten items and to fill in an anonymous data sheet on personal characteristics within $15 \mathrm{~min}$. They were informed that there were no correct or wrong answers except for item 10 .

Since the three survey samples differed in important characteristics such as participants' sex, profession or country of work, only descriptive data are presented. No statistical comparisons between subgroups were made.

The risk reduction for the primary endpoint in the UKPDS was $12 \%(95 \%$ CI $1-21 \%, p=0.029)$ with a number needed to treat for 10 years of $20(95 \%$ CI $10-500)$ [18]. In the conventional treatment group with a median $\mathrm{HbA}_{1 \mathrm{c}}$ of $7.9 \%$ over 10 years, 46 out of 100 patients had a primary endpoint, and in the intensified treatment group with a median $\mathrm{HbA}_{1 \mathrm{c}}$ of $7.0 \%, 41$ out of 100 patients suffered some kind of diabetes-related endpoint [18-20]. Responses for item 10 were rated as correct if estimates for natural frequencies did not deviate by more than $\pm 10 \%$ (41-51 out of 100 for conventional, 37-45 out of 100 for intensified therapy groups), figures were higher for the conventional treatment group than for the intensified treatment group, and the difference between the two groups was $>0 \leq 10$.

\section{Results}

Participation rate was $96 \%(299 / 312)$. A table with the characteristics of the three survey samples is available as ESM (ESM Table 1). In short, participants came from 20 different countries, although the JENA sample comprised almost solely participants from Germany. About half of all participants were nurses in diabetes (diabetes nurse specialists or educators), almost $40 \%$ were physicians. Two-thirds had a university degree. Place of work was 
evenly distributed between primary, secondary and tertiary care levels, though nurses were more likely to work at the secondary and tertiary care levels. On average, participants had worked for more than 10 years in clinical diabetology. The JENA group tended to have fewer years of practice in diabetes care.

Predefined assumptions were supported by the survey results (Table 1). Effects presented as changes in diabetes risk were considered more important than corresponding changes in glucose or glycosylated haemoglobin values (Table 1). More than two-thirds of respondents (188/277) considered a diabetes risk reduction of $57 \%$ (item 1) more important than corresponding changes in fasting plasma glucose values (mean difference $0.3 \mathrm{mmol} / \mathrm{l}$ ) (item 4). About $85 \%(234 / 274)$ viewed the difference in diabetes risk between $14 \%$ in the intervention group and $29 \%$ in the control group (item 8) to be more important than related changes in glycosylated haemoglobin values (6.0 versus $6.1 \%$; item 7). The impact of graphical framing becomes evident by the extent of variation between the ratings of the four presentation formats of the WHI study results (items 2 , 3, 5, 9; Table 1). About 73\% (202/278) rated the study effect on diabetes risk as more important when the slightly modified graph of the original publication was displayed (item 5) rather than a graph with a scale ranging from 0.0 to 1.0 (item 9).

Table 2 shows that only between 13 and $23 \%$ of subjects gave identical ratings for corresponding study results for the three diabetes prevention studies (items 1 and 4, items 7 and 8), and for items 2, 3,5 and 9 if at least three items were rated identically.

Item 10 (UKPDS) was filled in by only 53\% (158/299) of participants, and was answered correctly by only two people. Only 6\% (19/299) gave correct estimates for the event rate of the conventional treatment group, and $4 \%$ $(13 / 299)$ for the intensified treatment group. The difference between groups was within the range of correct estimates for $39 \%(61 / 158)$ of those with valid responses, $15 \%$
(23/158) reported a zero difference, whereas the rest substantially overestimated the intervention effect: in 34\% (54/158) estimates ranged from 10 to $30 \%$ points, and $13 \%$ (20/158) thought the difference was more than $30 \%$ points. Twenty-eight per cent (45/158) of respondents erroneously gave higher estimates for the conventional treatment group than for the intensified treatment group. Even if a wider range of responses had been considered as correct (30-55\% for the conventional treatment group, and $30-45 \%$ for the intensified treatment group) only six participants would have answered correctly.

\section{Discussion}

The present study uncovers a lack of understanding of the results of diabetes prevention studies by diabetes healthcare providers, who overestimate the benefit of preventive interventions when outcomes are presented as changes in diabetes risk rather than real metabolic changes.

The diabetes prevention studies included individuals with elevated fasting and post-load glucose concentrations who were already at the brink of diabetes [10-13]. Therefore, minimal differences in fasting plasma glucose of $0.3 \mathrm{mmol} / 1$ or $\mathrm{HbA}_{1 \mathrm{c}}$ values of $0.1 \%$ may relate to pronounced differences in the proportions of persons with a diagnosis of diabetes of $15 \%$ and diabetes risk reductions of more than $50 \%$ [16]. Small metabolic differences are magnified by transformation of continuous data into categorical data [16].

In some of the publications of the primary prevention studies, crucial metabolic data are not communicated or are difficult to extract [16]. The Finnish Diabetes Prevention Study included $\mathrm{HbA}_{1 \mathrm{c}}$ as a secondary outcome measure [21] but did not report results in the main publication [10]. Neither blood glucose nor $\mathrm{HbA}_{1 \mathrm{c}}$ values were reported in the core publication of the STOP-NIDDM Acarbose prevention study [13]. A recent systematic analysis by

Table 1 Ratings of study effects as important or very important in relation to format of presentation

\begin{tabular}{|c|c|c|c|c|}
\hline & FEND & PCDE & JENA & Total group \\
\hline \multicolumn{5}{|l|}{ Finnish Diabetes Prevention Study [10] } \\
\hline $57 \%$ risk reduction (item 1 ) & $132(91.0)$ & $38(88.4)$ & $78(80.4)$ & $248(87.0)$ \\
\hline Fasting glucose values (item 4) & $70(47.6)$ & $11(26.8)$ & $29(30.2)$ & $110(38.7)$ \\
\hline \multicolumn{5}{|l|}{ Diabetes Prevention Program [12] } \\
\hline $\mathrm{HbA}_{1 \mathrm{c}}$ values (item 7) & $37(25.2)$ & $6(14.3)$ & $9(9.6)$ & $52(18.4)$ \\
\hline Proportion of subjects with diabetes, high-risk group (item 8) & $132(93.0)$ & $37(92.5)$ & $86(91.5)$ & $255(92.4)$ \\
\hline \multicolumn{5}{|l|}{ Women's Health Initiative [17] } \\
\hline Probability of remaining free of diabetes, graphical scale from 0.0 to 1.0 (item 2) & $78(57.4)$ & $17(42.5)$ & $37(40.2)$ & $132(49.3)$ \\
\hline $21 \%$ risk reduction, hazard ratio, confidence intervals, $p$ value (item 3 ) & $118(82.5)$ & $37(90.3)$ & $69(72.6)$ & $224(80.3)$ \\
\hline Original graph, truncated scale (item 5) & $122(85.3)$ & $37(88.1)$ & $59(63.4)$ & $218(78.4)$ \\
\hline Diabetes risk, graphical scale from 0.0 to 1.0 (item 9) & $33(22.8)$ & $4(10.8)$ & $13(14.0)$ & $50(18.2)$ \\
\hline \multicolumn{5}{|l|}{ Unrelated to specific publication } \\
\hline Low-risk group, proportion of subjects with diabetes (item 6) & $104(73.2)$ & $26(63.4)$ & $44(46.8)$ & $174(62.8)$ \\
\hline
\end{tabular}

Values are numbers (percentages of respondents). Percentages may not add up to total number analysed due to missing values FEND Federation of European Nurses in Diabetes, Annual Conference 2005, PCDE Primary Care Diabetes Europe, Annual Conference 2005, JENA Postgraduate Course in Clinical Diabetology at the University of Jena, Germany, 2005 
Table 2 Participants with identical ratings for corresponding study results

\begin{tabular}{lllll}
\hline & FEND $(n=156)$ & PCDE $(n=45)$ & JENA $(n=98)$ & Total group $(n=299)$ \\
\hline Identical ratings for items 1 and 4 & $42(26.9)$ & $8(17.8)$ & $18(18.4)$ & $68(22.7)$ \\
Identical ratings for items 7 and 8 & $25(16.0)$ & $3(6.7)$ & $12(12.2)$ & $40(13.4)$ \\
Identical ratings for items 2, 3, 5 and 9 & $21(13.5)$ & $7(15.6)$ & $19(19.4)$ & $47(15.7)$ \\
\hline
\end{tabular}

Values are numbers (percentages)

adentical for at least three of the four items

FEND Federation of European Nurses in Diabetes, Annual Conference 2005, PCDE Primary Care Diabetes Europe, Annual Conference 2005, JENA Postgraduate Course in Clinical Diabetology at the University of Jena, Germany, 2005

Chan and Altman has found that incomplete reporting of outcomes within published articles of randomised trials is common and is associated with statistical non-significance [22]. Biased reporting and framing of data by researchers and authors may enhance misconceptions about treatment effects among users of study results.

The survey points to other possible sources of misunderstanding when reporting study results. Framing of graphical scales in publications is of concern. Identical study results of the WHI were rated as more important when the original scale was used rather than a scale ranging from 0.0 to 1.0. A truncated scale that is narrowed to the range of results visually magnifies the effects. In accordance with previous reports [23], participants considered graphically displayed effects more important when areas under the curves were large (item 2).

The impact of framing on understanding of clinical research data by diabetes healthcare professionals may be substantial. Only two to six of the 299 participants were able to correctly state the natural frequencies for the primary outcome measure of the UKPDS, a most popular study among diabetes healthcare professionals since its publication in 1998. About half of the participants did not answer at all, and of those who provided valid data, the effects of intensified therapy were substantially overestimated by $40 \%$ of participants. The UKPDS has been used as an example to demonstrate the significance of framing of data previously $[19,20]$. Therefore, results of reporting UKPDS data as natural frequencies could have been familiar to diabetes experts. Our findings may be interpreted as an indication that diabetes healthcare providers do not use or communicate natural frequencies of studies such as the UKPDS in their daily work with patients, students or colleagues.

The present study has limitations. The study population is a convenience sample and may not be representative of the whole community of healthcare workers in the field of diabetes. It consisted of a group of professionals particularly dedicated to continuous medical education, with longstanding experience in diabetes care and high academic levels on average - about half had participated in a postgraduate course in evidence-based medicine/nursing/ healthcare. Language barriers might have interfered with their understanding of the questionnaire.

From the results of this survey, we would urgently recommend that study results be presented in a format that can easily be understood by healthcare professionals.
Several reviews have summarised criteria for the communication of risk information to the public [3, 8, 24]. Research publication journals should insist on adequate data presentation by authors. This includes the use of scales with complete ranges of proportions from 0.0 to 1.0 and the presentation of results as natural frequencies and absolute risk reductions rather than relative risk reductions, particularly in the abstract. All outcome measures should be reported [22]. In addition, the research findings should be discussed with respect to their clinical relevance [16] Unbiased communication of research data is a prerequisite for participation of patients and the public in medical decision-making.

Acknowledgements This study was not funded, and no duality of interest exists for any of the authors.

\section{Appendix}

I. Mühlhauser, the principal investigator and guarantor of the study, conceived the research idea, designed the questionnaire, coordinated the study, interpreted the data, and wrote the paper. J. Kasper developed the database and undertook data analyses. G. Meyer contributed to the questionnaire and protocol design.

The Federation of European Nurses in Diabetes (FEND, London, UK) contributors were D. Cregan, A.M. Felton, and B. Hansen, all of whom helped design the questionnaire and contributed to pilot testing and data gathering.

All contributors commented on paper drafts.

\section{References}

1. Coulter A (1998) Evidence-based patient information. BMJ 317:225-226

2. Entwistle VA, Sheldon TA, Sowden A, Watt IS (1998) Evidence-informed patient choice. Int J Technol Assess Health Care 14:212-225

3. Edwards A, Elwyn G, Mulley A (2002) Explaining risks: turning numerical data into meaningful pictures. BMJ 324:827-830

4. Forrow L, Taylor WC, Arnold RM (1992) Absolutely relative: how research results are summarized can affect treatment decisions. Am J Med 92:121-124

5. Bucher HC, Weinbacher M, Gyr K (1994) Influence of method of reporting study results on decision of physicians to prescribe drugs to lower cholesterol concentration. BMJ 309:761-764 
6. Hoffrage U, Lindsey S, Hertwig R, Gigerenzer G (2000) Communicating statistical information. Science 290:2261-2262

7. Fahey T, Griffiths S, Peters TJ (1995) Evidence based purchasing: understanding results of clinical trials and systematic reviews. BMJ 311:1056-1060

8. Edwards A, Elwyn G, Covey J, Matthews E, Pill R (2001) Presenting risk information - a review of the effects of "framing" and other manipulation on patient outcomes. J Health Commun 6:61-82

9. Sackett DL (2002) The arrogance of preventive medicine. CMAJ 167:363-364

10. Tuomilehto J, Lindström J, Eriksson J-G et al (2001) Prevention of type 2 diabetes mellitus by changes in lifestyle among subjects with impaired glucose tolerance. NEJM 344:1343-1350

11. Lindstrom J, Louheranta A, Mannelin M et al (2003) The Finnish diabetes prevention study (DPS). Diabetes Care 26:3230-3236

12. Diabetes Prevention Program Research Group (2002) Reduction in the incidence of type 2 diabetes with lifestyle intervention or metformin. NEJM 346:393-403

13. Chiasson J-L, Josse RG, Gomis R, Hanefeld M, Karasik A, Laakso M for The STOP-NIDDM Trial Research Group (2002) Acarbose for prevention of type 2 diabetes mellitus: the STOPNIDDM randomised trial. Lancet 359:2072-2077

14. Diabetes UK. http://diabetes.org.uk, accessed Sep 62005

15. AG Prävention des Typ 2 Diabetes der DDG. http://www. diabetesprevention.de, accessed Sep 62005
16. Mühlhauser I (2002) Acarbose for type 2 diabetes prevention. Lancet 360:517

17. Margolis KL, Bonds DE, Rodabough RJ et al (2004) Effect of oestrogen plus progestin on the incidence of diabetes in postmenopausal women: results from the Women's Health Initiative Hormone Trial. Diabetologia 7:1175-1187

18. UK Prospective Diabetes Study (UKPDS) Group (1998) Intensive blood-glucose control with sulphonylureas or insulin compared with conventional treatment and risk of complications in patients with type 2 diabetes (UKPDS 33). Lancet 352:837-853

19. Berger M, Mühlhauser I (1999) Diabetes care and patient oriented outcomes. JAMA 281:1676-1679

20. Mühlhauser I, Berger M (2000) Evidence-based patient information in diabetes. Diabetic Medicine 17:823-829

21. Eriksson J, Lindström J, Valle T et al (1999) Prevention of Type II diabetes in subjects with impaired glucose tolerance: the Diabetes Prevention Study (DPS) in Finland. Study design and 1-year interim report on the feasibility of the lifestyle intervention programme. Diabetologia 42:793-801

22. Chan AW, Altman DG (2005) Identifying outcome reporting bias in randomised trials on PubMed: review of publications and surveys of authors. BMJ 330:753-756

23. Lipkus IM, Hollands JG (1999) The visual communication of risk. Monogr Natl Cancer Inst 25:149-156

24. Steckelberg A, Berger B, Köpke S, Heesen C, Mühlhauser I (2005) Kriterien für evidenzbasierte Patienteninformationen (Criteria for evidence-based patient information). Z Arztl Fortbild Qualitatssich 99:343-351 